\title{
Bayesian Hierarchical Estimation and Impact Analysis of Generalized Production Functions
}

\author{
Xinping Yang ${ }^{1}$, Wei Zheng ${ }^{1}$, Yunyuan Yang ${ }^{2}$, Yanmei $\mathbf{L i}^{1,}$, \\ ${ }^{1}$ School of Mathematics and Statistics, Chuxiong Normal University, Chuxiong, China \\ ${ }^{2}$ School of Geography and Tourist Management, Chuxiong Normal University, Chuxiong, China
}

Email address:

lym@cxtc.edu.cn (Yanmei Li)

${ }^{*}$ Corresponding author

\section{To cite this article:}

Xinping Yang, Wei Zheng, Yunyuan Yang, Yanmei Li. Bayesian Hierarchical Estimation and Impact Analysis of Generalized Production Functions. American Journal of Theoretical and Applied Statistics. Vol. 10, No. 2, 2021, pp. 122-128. doi: 10.11648/j.ajtas.20211002.14

Received: March 19, 2021; Accepted: March 30, 2021; Published: April 12, 2021

\begin{abstract}
Bayesian model is constructed to estimate the missing data of capital stock, and the impact of three industrial structures on the tertiary industry is discussed. The results show that: the maximum MC error with missing data on capital stock estimated by Bayesian is 0.4963 , the maximum MC error of production function estimated by Bayesian stratation is 0.3276 , the standard deviation is 0.0890 and the accuracy is high; from 1993 to 2018, the sum of capital output elasticity and labor output elasticity in the tertiary industry in Yunnan Province was greater than 1, and the scale compensation was increasing; the level of technological progress, the growth rate of all factors, the elasticity of capital output and the elasticity of labor output were all close to stable, and the ranges of changes were $0.2714-0.3252,-0.0680-0.0390,0.5615-0.5858$ and $0.4522-0.4784$, respectively; the elasticity of capital output was greater than that of labor force output, and the tertiary industry in Yunnan Province was more dependent on the elasticity of capital output.
\end{abstract}

Keywords: Generalized Production Function, Capital Stock, Non-random Missing, Gibbs Sampling, Impact Analysis

\section{Introduction}

In recent years, the methods of industrial research based on C-D production function mainly include: (1) regression after the linearization of model of C-D production function. Based on the data of the tertiary industry in Shandong Province, Huang estimated the parameters of this function on the basis of regression, and put forward corresponding suggestions. Based on the production function, [1] wang made an empirical study on the industrial development of Anhui Province by linear regression method. [2] Feng et al estimated the parameters of the generalized C-D production function by regression method based on inner Mongolia service data, and put forward some suggestions for the development of local service industry. [3] (2) Analysis by using the Bayesian method. Wang et al used the Bayesian method to estimate the alternative elasticity of CES production functions based on Shanghai data. [4] Zeng et al amended and renewed the parameters of the generalized C-D production function based on the Bayesian panel model. [5] (3) Analysis by using the horizontal model. Shi et al studied C-D production functions and other one-equation economic models by two-level models. [6] In the above study, the regression method used to estimate the parameters of the production function (e.g. [1-3]) must rely on a harsher assumption: the share of capital remains the same over a longer period of time. [5] By using the Bayesian method, making full use of a priori and data information could weaken or even eliminate this hypothesis, and the estimation accuracy of relevant parameters (e.g. [4-5]) could be improved by simulating a large number of post-test samples. At present, papers are rare that have quantitative study of the impact of three industrial structures on the relevant elements of the tertiary industry in a certain region and fully results of calculation. The more important result was that Liuet al put forward a two-level model of the impact of variable coefficient model on production scale and factor efficiency, and the study used the method of least squares method to complete the parameter estimation, [7] but the accuracy of the estimate needed to be improved.

In practice, the capital stock in the production function is a difficult point to calculate. Most researchers use Goldsmith's (1951) permanency intransifration method (PIM) to estimate, which has two estimation models: linear efficiency decline and geometric 
efficiency decline. In addition, different researchers used different depreciation rates and different methods of estimation, and would get different results, resulting in a large error of calculation in capital stock or difficulty in correct. Furthermore, the lack of regional statistics can sometimes make the capital stock uncalculable. Generally, the lack of data is common in economic research. Rubin and Molenberghs divided the missing data into three categories. They are: Completely Random Missing (MCAR), Random Missing (MAR), and Non-Random Missing (MNAR). [8-9] For the processing of missing data, the simple method is to replace it with the average or subjectively appropriate value, or even ignore it, which is simple and rough, and the error is larger. A better approach is to choose the appropriate model for simulation design according to the formation mechanism of data missing.

In connection with the shortcomings in the study above-mentioned, the Bayesian model is established to make a reasonable estimate of the missing data of capital stock, taking the three industrial data of Yunnan Province from 1990 to 2018 as an example. By using the Bayesian model of lamination, the total factor growth rate (TFP), capital output elasticity and labor output elasticity in the generalized C-D production function are estimated, and the evaluation and diagnosis of the model are completed. The influence of the three industrial structures on the growth rate of the whole factor, the elasticity of capital output and the elasticity of labor output is further discussed, and the results of the study have some guiding significance to the formulation of the tertiary industry development policy in Yunnan Province.

\section{The Bayesian Model of Lamination of the Production Function}

\subsection{Generalized Production Functions and Linearization}

The generalized C-D production functions can generally be expressed as: [3]

$$
Y_{\mathrm{t}}=A e^{\lambda t} L_{t}^{\beta} K_{t}^{\alpha} e_{t}
$$

Where $A$ represents the level of technological progress, $Y_{t}, K_{t}, L_{t}$ and $A e^{\lambda t}$ represent the actual output of the tertiary industry, the capital stock, the labor input of the tertiary industry and The level of comprehensive scientific and technological progress respectively in the $\mathrm{t}$ year, and $\lambda$ is the total factor productivity or TFP growth rate [3]. $\alpha(0<\alpha<1)$ and $\beta(0<\beta<1)$ are called the elastic coefficient of capital output and labor output respectively. Taking logarithm on both sides of (1) yields: $\quad \ln \mathrm{Y}_{\mathrm{t}}=\ln \mathrm{A}+\lambda \mathrm{t}+\alpha \ln \mathrm{K}_{\mathrm{t}}+\beta \ln \mathrm{L}_{\mathrm{t}}+\ln \mathrm{e}_{\mathrm{t}}$. Denote $y_{t}=\ln Y_{t}, k_{t}=\ln K_{t}, l_{t}=\ln L_{t}, \varepsilon_{t}=\ln e_{t}$ and we get:

$$
y_{t}=a+\lambda t+\alpha k_{t}+\beta l_{t}+\varepsilon_{t}
$$

\subsection{Bayesian Hierarchical Model and Gibbs Sampling}

\subsubsection{Bayesian Hierarchical Model}

Wei Liu et al discussed the model (2) by using the average of the proportion $\bar{x}_{j}, \mathrm{j}=1: \mathrm{m}$ as covariaials of the parameters $a, \lambda, \alpha, \beta$ of m-sub-industrial structure in a certain period of time. On the basis of above study, by using the Bayesian method, the parameters are set in two layers for the model (2), the appropriate distribution is specified, the parameters $a, \lambda, \alpha, \beta$ are expressed by the linear function of the industrial structure index of a region, and the influence of the industrial structure of a region on the output scale and the parameters of economic significance is quantitatively studied.

Set the random error $\varepsilon_{t} \sim N\left(0, \sigma^{2}\right)$ in the model (2), specify the appropriate distribution for each layered parameter, and establish the Bayesian hierarchical model as:

$$
\left\{\begin{array}{cc}
y_{t} \sim N\left(\mu_{t}, \tau\right), \quad \mu_{t}=a+\lambda t+\alpha k_{t}+\beta l_{t}, \quad \tau=1 / \sigma^{2} \\
a \sim N\left(\sum_{j=1}^{m} a_{j} x_{j}, \tau_{1}\right), \quad \tau_{1}=1 / s_{1}^{2} \\
\lambda \sim N\left(\sum_{t=1}^{m} b_{j} x_{j}, \tau_{2}\right), \quad \tau_{2}=1 / s_{2}^{2} \\
\alpha \sim N\left(\sum_{j=1}^{m} c_{j} x_{j}, \tau_{3}\right), \quad \tau_{3}=1 / s_{3}^{2} \\
\beta \sim N\left(\sum_{j=1}^{m} d_{j} x_{j}, \tau_{4}\right), \quad \tau_{4}=1 / s_{4}^{2}
\end{array}\right.
$$

where $\mu_{t}, \sigma^{2}$ are model parameters and $\theta^{\mathrm{T}}=(a, \lambda, \alpha, \beta)$ is the first layering hyperparameter of the model, $\vartheta^{T}=\left(a_{1}, \ldots, a_{m}, s_{1}^{2}, b_{1}, \ldots, b_{m}, s_{2}^{2}, c_{1}, \ldots, c_{m}, s_{3}^{2}, d_{1}, \ldots, d_{m}, s_{4}^{2}\right)$ is the second layering hyperparameter of the model.

What is concerned here is the estimations of first layering superparameter and second layering superparameter. The first layering superparameter is the parameter of economic significance in the generalized C-D production function, and the second layering hyperparameter reveals the influences of the level of technological progress, total factor productivity, capital output elasticity and labor output elasticity in C-D production function in the three industrial structures. $\alpha+\beta>$ 1 indicates an increase in scale compensation and, conversely, a decrease in scale compensation. [6]

For $a_{j}, b_{j}, c_{j}, d_{j}, j=1: m$ in the second layering hyperparameters, there is no prior information available, and according to the proposition of Lunn D, et al (2013) [10-11], specify the normal distribution with large variance $N\left(0,10^{4}\right)$ as their prior distribution and choose model variance $\sigma^{2}$ and the uniform distribution $U(0,100)$ of two-tier variance parameters $s_{i}^{2}, i=1: 4$ for their priori distributions respectively. According to Bayesian theory, the post-validation distribution of the super-parameters is

$$
\begin{gathered}
\mathrm{p}\left(\vartheta \mid y_{t}, k_{t}, l_{t}, x_{j}, t\right) \propto \tau^{\frac{n}{2}}\left(\tau_{1} \tau_{2} \tau_{3} \tau_{4}\right)^{\frac{1}{2}} \exp \left(-\frac{\tau}{2} \sum_{\mathrm{t}=1}^{n}\left(y_{t}-\left(a+\lambda t+\alpha k_{t}+\beta l_{t}\right)\right)^{2}\right) \times \\
\exp \left(-\frac{\tau_{1}}{2}\left(a-\sum_{j=1}^{m} a_{j} x_{j}\right)^{2}-\frac{\tau_{2}}{2} \times\left(\lambda-\sum_{j=1}^{m} b_{j} x_{j}\right)^{2}-\frac{\tau_{3}}{2}\left(\alpha-\sum_{j=1}^{m} c_{j} x_{j}\right)^{2}-\frac{\tau_{4}}{2}\left(\beta-\sum_{j=1}^{m} d_{j} x_{j}\right)^{2}-\frac{1}{2 \times 10^{4}} \sum_{j=1}^{m}\left(a_{j}^{2}+b_{j}^{2}+c_{j}^{2}+d_{j}^{2}\right)\right)
\end{gathered}
$$

\subsubsection{Gibbs Sampling}

After formula finishing (4), it is found that under the condition that other components are known, the full condition distributions of other parameters are normal, 
except that the full condition distribution of variance parameters is inverse Gamma distribution. After the 1-step Gibbs sampling is completed, when carrying out step $1+1$, the updates completed in step $1+1$ are retained. The unfinished updates remain the values of step 1 , and the corresponding components are updated by the normal distribution and the inverse Gamma distribution. After the due number of iterations is reached, each MC chain appears in a convergent state. Stops iteration updates, obtains a post-test sample of each parameter, and completes the Bayesian hierarchical estimation of the parameters by using the MCMC method.

\section{Analysis of the Essential Factors Affecting the Tertiary Industry in Yunnan Province}

\subsection{The Source of Data and Absence of Data}

\subsubsection{Source of Data and Deficiency of Index of Price}

Collect the output value of the tertiary industry (se, in 100 million yuan), the total amount of fixed asset formation (fix, unit: billion yuan), the number of employees in the tertiary industry at the end of the year (see, in 10,000), the price index (ip) and capital Stock data (sc) in Yunnan Province from 1990 to 2018 as sample data (the first three indicators were from the 2019 Yunnan Provincial Statistical Yearbook, the latest two indicators were derived from Liu da-peng [12]), in which the price index and capital stock data in 2014-208 are missing and belong to non-random missing (MNAR), and is estimated by establishing a suitable model.

Comparing the price indices after taking logarithm for 1990-2013, it was found that the values for 1990-1992 were relatively small, with the remaining values between 5 and 6 , showing a linear slow growth pattern. As the lag effect of economic data does not exceed 2 periods, the three values are removed, and the self-regression model (b) of all data is established at the same time as the linear model (a) of time $t$ is established. Their parameter estimates and statistics are seen in Table 1 respectively, and the missing price index for 2014-2018 is estimated by using the corresponding model. The results can be found in Tables 1 and 2 .

Table 1. Parameter estimation and statistics of linear model and autoregressive model of logarithmic price index.

\begin{tabular}{|c|c|c|c|c|c|c|}
\hline \multirow{2}{*}{ Model $(y=\log (i p))$} & \multirow{2}{*}{$R^{2}$} & \multirow{2}{*}{ adejusted $R^{2}$} & \multirow{2}{*}{ p-value } & \multicolumn{2}{|l|}{$P_{\mathrm{r}}(>|t|)$} & \multirow{2}{*}{ deviance } \\
\hline & & & & Constant & One-time item & \\
\hline Model (a): $y_{t}=5.201876+0.029062 t$ & 0.9766 & 0.9754 & $2.2 \mathrm{e}-16$ & 0.0000 & 0.0001 & 0.015543 \\
\hline Model $(b): y_{t}=0.864224+0.84927 y_{t-1}$ & 0.9682 & 0.9667 & 0.0000 & 0.0000 & 0.0000 & 0.051746 \\
\hline
\end{tabular}

Note: (1) $P_{\mathrm{r}}(>|t|)$ represents the companion probability of the two-sided t-test. (2) The statistical evaluation index of the two models shows that the model fits better.

Table 2. Estimation of logarithmic missing value of price index in model (a) and model (b) respectively.

\begin{tabular}{|c|c|c|c|c|c|}
\hline Logarithm of index of price & Estimation in 2014 & Estimation in 2015 & Estimation in 2016 & Estimation in 2017 & Estimation in 2018 \\
\hline Forcast of (a) & 5.695922 & 5.724983 & 5.754045 & 5.783106 & 5.812168 \\
\hline Forcast of (b) & 5.787222 & 5.781783 & 5.777161 & 5.773234 & 5.769897 \\
\hline
\end{tabular}

\subsubsection{Estimate of the Lack of Capital Stock}

The Bayseian model (5) is established by using the estimate of the missing logarithm price index in Table 2 together with the logarithm scale of the Yunnan Provincial Price Index [12] for 1990-2013 as complete data. After the parameter estimation is completed, the missing capital stock (missing response variables) for each year in 2014-2018 is estimated by using model (5) to prepare for the estimation of the generalized production function in the following text.

$$
\left\{\begin{array}{c}
\mathrm{sc}_{i} \sim N\left(\mu_{i}, \tau\right) \quad \tau=\frac{1}{\sigma^{2}} \\
\mu_{i}=\beta_{0}+\beta_{1} f i x_{i}+\beta_{2} \log \left(i p_{i}\right)
\end{array}\right.
$$

For parameters $\beta_{i}, i=1: 3$ and $\sigma^{2}$, we choose the priori method in the second part, and specify normal distribution $N\left(0,10^{4}\right)$ and even distribution $U(0,100)$ as their priori distributions, respectively. Let initial values $\beta_{0}=0.8, \beta_{1}=0.9, \beta_{2}=4.4, \sigma^{2}=99$, and other initial values are automatically generated by the program. In order to reduce the self-correlation of the post-test sample and ensure the convergence of the Mars chain (MC chain), the interval sampling step is 100 and the combustion period is 5000. After obtaining 9 post-test samples ( $9 \mathrm{MC}$ chains) of the parameters to be evaluated through the $10^{5}$ sampling update, for each parameter's post-test samples, throw away 4999 samples from the combustion period and complete the Bayesian inference of each parameter with the remaining 95001 samples, and the corresponding parameter estimates are found in Table 3 . In Table 3, the mean estimates (Mean), median estimates (Median), and 95\% confidence interval CI for the median values ( $2.5 \% \mathrm{ql}, 97.5 \% \mathrm{qu})$, standard deviation (sd), and $\mathrm{MC}$ error (MC error) are given. 
Table 3. Bayesian estimation and statistical summary of missing capital stock.

\begin{tabular}{|c|c|c|c|c|c|c|c|c|c|c|c|c|c|}
\hline \multicolumn{7}{|c|}{ Model $(a)$} & \multicolumn{7}{|c|}{ Model (b) } \\
\hline node & Mean & sd & MCerror & $2.50 \% q \mathrm{l}$ & median & $97.50 \% q u$ & node & Mean & sd & MCerror & $2.50 \% \mathrm{ql}$ & median & $97.50 \% q u$ \\
\hline$\beta_{0}$ & 0.8312 & 0.9984 & 0.0030 & -1.1390 & 0.8309 & 2.7960 & $\beta_{0}$ & 0.8286 & 0.9988 & 0.0035 & -1.1320 & 0.8263 & 2.7930 \\
\hline$\beta_{1}$ & 0.8896 & 0.0059 & $1.88 \mathrm{e}-05$ & 0.8778 & 0.8896 & 0.9013 & $\beta_{1}$ & 0.8896 & 0.0059 & $1.91 \mathrm{e}-05$ & 0.8778 & 0.8896 & 0.9013 \\
\hline$\beta_{2}$ & 4.4790 & 0.9806 & 0.0034 & 2.5670 & 4.4780 & 6.4080 & $\beta_{2}$ & 4.4800 & 0.9788 & 0.0033 & 2.5590 & 4.4790 & 6.3950 \\
\hline $\mathrm{sc}_{25}$ & 10260 & 120.90 & 0.3709 & 10020 & 10260 & 10490 & $\mathrm{sc}_{25}$ & 10260 & 121.00 & 0.3807 & 10020 & 10260 & 10490 \\
\hline $\mathrm{sc}_{26}$ & 12040 & 128.20 & 0.3789 & 11790 & 12040 & 12290 & $\mathrm{sc}_{26}$ & 12040 & 128.50 & 0.3999 & 11780 & 12040 & 12290 \\
\hline $\mathrm{Sc}_{27}$ & 14370 & 137.90 & 0.4362 & 14100 & 14370 & 14640 & $\mathrm{sc}_{27}$ & 14370 & 138.10 & 0.4445 & 14100 & 14370 & 14640 \\
\hline $\mathrm{sc}_{28}$ & 16870 & 150.30 & 0.4863 & 16580 & 16870 & 17170 & $\mathrm{sc}_{28}$ & 16870 & 150.40 & 0.4666 & 16580 & 16870 & 17170 \\
\hline $\mathrm{sc}_{29}$ & 18780 & 160.10 & 0.4963 & 18460 & 18780 & 19090 & $\mathrm{sc}_{29}$ & 18780 & 160.30 & 0.5264 & 18460 & 18770 & 19090 \\
\hline$\sigma$ & 99.850 & 0.1508 & $4.94 \mathrm{e}-04$ & 99.440 & 99.890 & 100.00 & $\sigma$ & 99.850 & 0.1501 & $4.82 \mathrm{e}-04$ & 99.450 & 99.890 & 100.00 \\
\hline
\end{tabular}

On the basis of completing the logarithm estimate of the missing price index for model (a) and (b), the Bayesian estimate of the missing capital stock obtained by model (5) (including the estimate of the mean, $95 \%$ of the confidence interval estimate) is almost the same. The rest estimates that have differences are expressed in boldface in Table 3. There are fewer estimates of differences in the table, and the differences do not occur until the second place after the decimal point. Other differences of statistic in judging the estimation accuracy of model parameters are also small and only differ in the second place after the decimal points. The largest (max. MC error/sd) $\times$ $100 \%$ has a value of $0.3100 \%$ in sub-table to the left, which is less than the corresponding value $(0.3280 \%)$ of the sub-table to the right of Table 3 , and both of them are less than $10 \%$. Table 3 indicates that the missing values are estimated with model (a) with higher accuracy. [11]

\subsection{Hierarchical Estimation and Impact Analysis of the Production Functions of the Tertiary Industry in Yunnan Province}

\subsubsection{Bayesian Hierarchical Estimation and Statistical Diagnosis}

After completing the estimate of the missing capital stock and according to the model (3), the Bayesian hierarchical model to study the impact of industrial structure on the production scale, technological progress, total factor productivity and output elasticity of the tertiary industry was established by using the increment of the tertiary industry, the data of the three industrial structures, the capital stock and the number of employees at the end of the year in the tertiary industry in Yunnan Province from 1990 to 2018 as the sample.

Table 4. Bayseian stratified parameter estimation and statistics summary of generalized C-D production function.

\begin{tabular}{|c|c|c|c|c|c|c|}
\hline node & Mean & sd & MC_error & $2.50 \% q \mathrm{l}$ & median & $97.50 \%$ qu \\
\hline$a_{1}$ & -0.5883 & 95.68 & 0.3262 & -188.7 & -0.3812 & 186.6 \\
\hline$a_{2}$ & -1.5400 & 82.90 & 0.2903 & -164.1 & -1.4770 & 162.3 \\
\hline$a_{3}$ & -1.3160 & 85.72 & 0.2924 & -169.1 & -1.3170 & 166.1 \\
\hline$b_{1}$ & 0.2043 & 95.79 & 0.3276 & -188.8 & 0.3769 & 187.7 \\
\hline$b_{2}$ & -0.1455 & 82.83 & 0.2719 & -163.9 & -0.2463 & 162.1 \\
\hline$b_{3}$ & -0.0847 & 85.48 & 0.2770 & -167.9 & 0.005195 & 168.1 \\
\hline$c_{1}$ & 0.4663 & 96.08 & 0.3029 & -187.6 & 0.1398 & 189.6 \\
\hline$c_{2}$ & 0.7704 & 82.91 & 0.2708 & -162.6 & 0.4841 & 164.2 \\
\hline$c_{3}$ & 0.4302 & 85.66 & 0.2652 & -167.1 & 0.6063 & 168.9 \\
\hline$d_{1}$ & 0.1703 & 95.87 & 0.3011 & -188.0 & 0.1138 & 189.7 \\
\hline$d_{2}$ & 0.7209 & 82.72 & 0.2912 & -161.0 & 0.8071 & 163.4 \\
\hline$d_{3}$ & 0.3262 & 85.41 & 0.3018 & -166.9 & 0.5599 & 167.4 \\
\hline$s_{1}$ & 44.160 & 28.22 & 0.09252 & 1.956 & 41.19 & 96.27 \\
\hline$s_{2}$ & 44.080 & 28.27 & 0.09515 & 1.963 & 41.10 & 96.41 \\
\hline$S_{3}$ & 44.010 & 28.24 & 0.09164 & 1.963 & 41.10 & 96.22 \\
\hline$s_{4}$ & 44.012 & 28.23 & 0.09060 & 1.962 & 41.05 & 96.15 \\
\hline$\sigma$ & 0.08903 & 0.01343 & $6.32 \mathrm{E}-05$ & 0.06741 & 0.08746 & 0.1197 \\
\hline
\end{tabular}

In order to obtain the post-test sample of each parameter of the model (3), we choose 700 and 5000 as the interval sampling step and the combustion period, respectively. The post-test samples of 17 parameters to be evaluated are obtained by using the orderly relaxation algorithm and $10^{5}$ Gibbs iterative sampling. Discard the samples from the previous 4999 combustion periods and use the remaining 95001 samples to complete the Bayesian inference of the second layering hyper-parameter, and the parameter estimates and statistics results are summarized in Table 4.

The parameter $\sigma^{2}$ of the standard deviation of model (3) is estimated to be $\hat{\sigma}=0.0890$, and the MC error is $6.32 \mathrm{E}-05$, which are very small, indicating that the accuracy of Bayesian estimation of the production function is high. The largest $\mathrm{MC}$ error in Table 4 (max. MC error) is 0.3276 , with a value ( $\max$. $M C$ error $/ \mathrm{sd}) \times 100 \%) 3.42 \%<10 \%$, further indicating that the parameters of model (3) are estimated with high accuracyand the model is reasonable. [13-14] In order to diagnose the convergence of the post-test samples and relatedness of model (3), the History-strace-plot graph, the nuclear density estimation graph and the autocorrelation graph (see figure 1) are used as examples of the post-test samples of parameters $a_{1}$ and $s_{1}$, (the graphs of the other 48 parameters are omitted). The results show that the MC chain converges, the nuclear density estimate of standard deviation is not normal distribution and the nuclear densities of other parameters are 
estimated to be normal distribution. If the lag period is greater than 1 , the coefficient of autocorrelation is close to 0 , and the
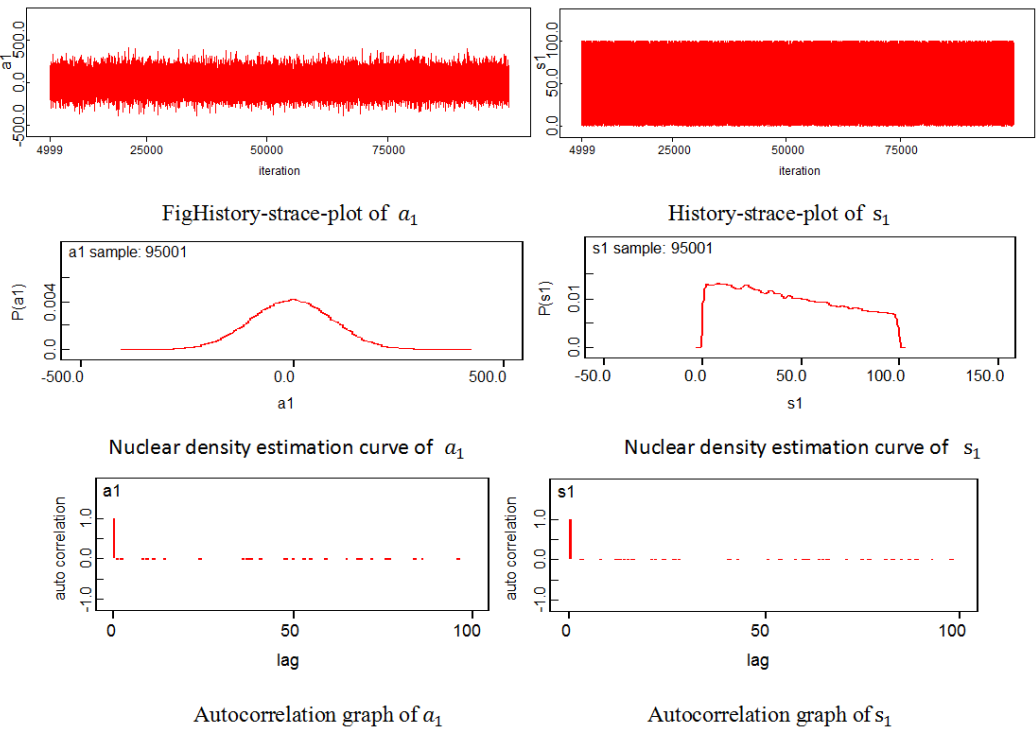

Figure 1. History strace plot, kernel density estimation curve and autocorrelation graph of $a_{1}$ and $s_{1}$

\subsubsection{Analysis of the impact of production functions on the tertiary industry in Yunnan Province}

The corresponding parameter estimates in Table 4 are substituted into the model (3) and reduced to the initial model before the mathematical simplification, and the generalized C-D production functions of technological progress level, total factor productivity, capital output elasticity and labor output elasticity affected by the three industrial structures are estimated as:

$$
\left\{\begin{array}{c}
\hat{Y}_{t}=A e^{\hat{\lambda} t} L_{t}^{\hat{\beta}} K_{t}^{\hat{\alpha}} \\
\hat{A}=0.555270^{x_{1}} 0.214381^{x_{2}} 0.268206^{x_{3}} \\
\hat{\lambda}=0.2043 x_{1}-0.1455 x_{2}-0.0847 x_{3} \\
\hat{\alpha}=0.4463 x_{1}+0.7704 x_{2}+0.4302 x_{3} \\
\hat{\beta}=0.1703 x_{1}+0.7209 x_{2}+0.3262 x_{3}
\end{array}\right.
$$

where $x_{j}, \mathrm{j}=1,2,3$ express the mean value of the proportion of three industrial structure in a time interval.

In model (6), $n=1$ expresses the estimate of production function of base period. If $n>1$, the formulae (6) express the estimate of production function in tertiary industry affected by three industrial structures in $n$ years. If the average of the proportion of three industrial structures is changed to the proportion $x_{t j}, t=1: n, j=1,2,3$ of three industrial structures of $t$ year, the trend change curve of technological progress level, total factor productivity, capital output elasticity and labor output elasticity can be obtained by drawing. Substitution of the average of the proportion of three industrial structures into (6) gives the corresponding value of the level of technological progress, total factor productivity, capital output elasticity and labor output elasticity in 1990-2018, and we can plot the curves. (For the sake of drawing convenience, the corresponding parameters are capital full-name, Figure 2). [15]

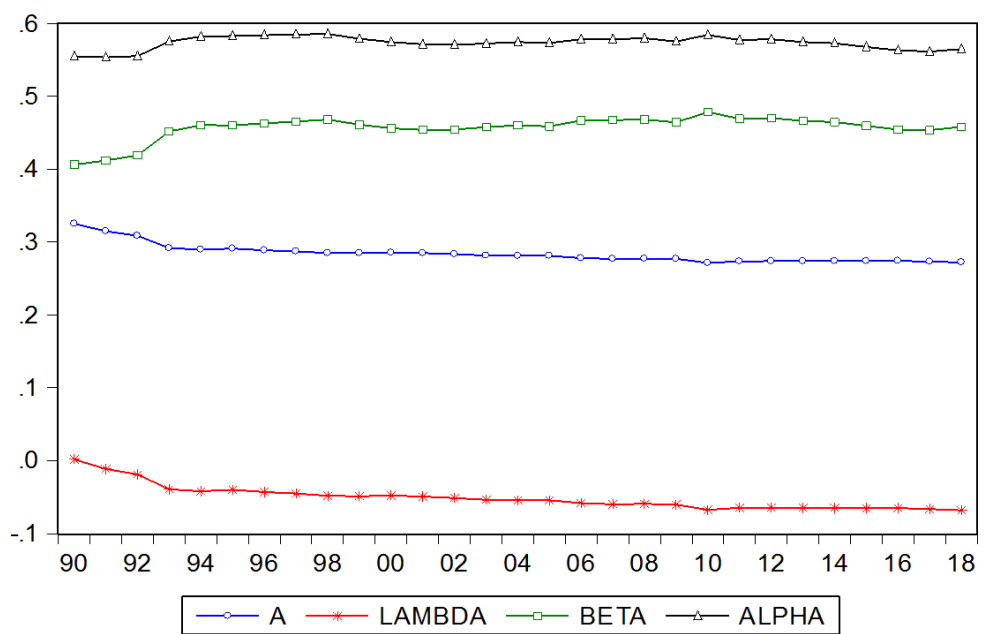

Figure 2. Change curve of technological progress level, total factor productivity, capital output elasticity and labor output elasticity in Yunnan Province from 1990 to 2018 . 


\section{Conclusion}

Firstly, the non-random missing capital stock data were estimated by using the three models ((a), (b), (5)) and data on three industrial output values, capital stocks, price indices and the number of people employed in the tertiary industry at the end of the year in Yunnan Province from 1990 to 2018. Secondly, the Bayesian stratation model is constructed, which studies the impact of Yunnan's industrial structure on production scale, factor efficiency and output elasticity, we complete the estimation of the production function of the tertiary industry in Yunnan Province, and draw the following conclusions:

(1) The established model achieves the deserved predictive accuracy: the maximum MC error of the missing value prediction model (5) is 0.4963 , the maximum MC error of the production function estimation (6) is 0.3276 , the standard deviation is 0.0890 , the corresponding $\mathrm{MC}$ chain of each parameter converges to its own post-test distribution, and the sample is an independent sample.

(2) When $\mathrm{n}=1$, the proportions of three industrial structures in base period (the year 1990) were $x_{1}=$ $0.3722, x_{2}=0.3494, x_{3}=0.2784$ Level of technological progress was estimated at $\widehat{A}=0.3252$. Estimates of total factor productivity, capital output elasticity and labor output elasticity were $\hat{\lambda}=0.0002, \hat{\alpha}=0.5550$ and $\hat{\beta}=0.4061$, respectively. The coefficients in model (6) reflected the influence of three industrial structures of the base period on the level of technological progress, the total factor productivity, the elasticity of capital output and the elasticity of labor output. Specifically speaking: the proportion of the first industry in Yunnan Province increased by $1 \%$, the growth rate of the whole factor increased by 0.2043 , the elasticity of capital output increased by 0.4463 , and the elasticity of labor output increased by 0.1703 . The proportion of secondary industry increased by $1 \%$, the total factor growth rate decreased by 0.1455 , the elasticity of capital output increased by 0.7704 , and the elasticity of labor output increased by 0.7209 . The proportion of tertiary industry increased by $1 \%$, the growth rate of total factor decreased by 0.0847 , the elasticity of capital output increased by 0.4302 , and the elasticity of labor output increased by 0.3262 . The elasticity of capital output is greater than that of labor rate, and the dependence of the tertiary industry on the elasticity of capital output is greater than that of the elasticity of labor output. The total factor productivity is close to $0(0.0002)$, which shows that the productivity improvement and industrial upgrading of the tertiary industry in Yunnan Province are not obvious, and the main factors that constitute the total factor productivity, such as scientific and technological progress, management level and investment environment, need to be improved from the whole.

(3) When $n=29$, substituting the proportion of three industrial structures $x_{i j}$ into model (6), we get the estimates of the level of technological progress, total factor productivity, capital output elasticity and labor output elasticity of Yunnan
Province from 1990 to 2018 year by year. The results show that the level of technological progress and the growth rate of all factors in Yunnan Province from 1990 to 2018 appeared a downward trend with a small scope. During 1990-1993, the values of $\alpha+\beta$ are $0.9611,0.9656$ and 0.9743 respectively, which were less than 1 , and this indicated that the scale of compensation was decreasing year by year. After 1993, $\alpha+\beta$ are larger than 1 , which means the scale compensation is increasing year by year, the level of technological progress, the growth rate of the whole factor, the elasticity of capital output and the elasticity of labor output have basically stabilized in 29 years (see Figure 2), and the ranges of change are smaller: $0.2714-0.3252,-0.0680-0.0390,0.5615-0.5858$, $0.4522-0.4784$. It shows that in the process of the development of the tertiary industry in Yunnan Province, the scientific and technological progress, management level and external investment environment need to be strengthened. While increasing the investment in fixed assets, we should improve the level of science and technology and management, enhance the external investment environment, strengthen the high integration of the tertiary industry with the first industry and the secondary industry, and promote the development of Yunnan's economy as a whole.

\section{Funding}

This research was funded by "Analysis of the Influence Mechanism of Modern Service Industry in Yunnan Province Based on Bayes Method" on the Project of Yunnan University Joint Fund. (2017FH001-068)

\section{References}

[1] Hang shuoshuo. An empirical analysis of the development of the tertiary industry in Shandong Province-Based on Cobb-Douglas production function [J]. Caixun, 2017, (4): 107-109.

[2] Wang ziqing. An empirical analysis of the development of tertiary industry in Anhui Province Based on production function [J]. Modern enterprise culture, 2012, (7): 125-127.

[3] Feng liying, Feng ruiqin, Li haixia. The production efficiency measurement of Inner Mongolia service industry Based on Cobb-Douglas production function [J]. Journal of Inner Mongolia University of Finance and Economics, 2015, 2 (13): 9-14.

[4] Wang jun, Liu lang-juan. Estimation of Elasticity of Substitution in CES function of major Industries in Shanghai [J]. Shanghai Journal of Economics, 2012, (5): 106-112.

[5] Zeng zhao-fa, Mi xian-hua. The Estimation and empirical of extended C-D production function based on Bayesian panel model [J], Statistic \& Iformation Forum, 2014, 7 (29): 29-34.

[6] Shi lei, Xiang qi-feng, Cheng fei. Multilevel model and its application in Economics [M]. China science press, 2013.

[7] Liu wei, Li shao-rong. Industrial structure and economic growth [J]. China industrial economy, 2002, (5): 1-8. 
[8] Rubin D B. Inference and missing data [J]. Biometrika, 1976, 63 (3): 581-592.

[9] Molenberghs, Kenward M G. Missing data in clinical stydies [M]. Joha Wiley \& Sons, New York, 2008.

[10] Daniels M J, Hogan J W. Missing data in longitudinal studies: Strategies for Bayesian modeling and sensitivity analtsis $[\mathrm{M}]$. Chapman \& Hall/CRC, Boca Raton, FL, 2008.

[11] Lunn D, Jackson Christopher, Best Nicky, et al. A practical introduction to bayesian Analysis [M]. Chapman \& Hall/CRC Press, 2013.
[12] Liu da-peng. Re-estimation of capital stock: Yunnan case [J]. 2016, (7): 244-245.

[13] Ioannis Ntzoufras. Bayesian modeling using WinBUGS [M]. Wiley, Hoboken, NJ, 2008.

[14] Yang yun-yuan, Yang xin-ping, Zhang jie. Bayesian estimation of domestic covid-19 epidemic data based on Dirichlet model [J]. Journal of Sichuan normal university (natural science). 2020, 11 (43): 838-846.

[15] Li ya, Li zhi-peng. Econometric analysis and Eviews [M]. China social sciences press, 2017. 